\title{
SUPERCHARACTERS AND PATTERN SUBGROUPS IN THE UPPER TRIANGULAR GROUPS
}

\author{
TUNG LE
}

\begin{abstract}
Let $U_{n}(q)$ denote the upper triangular group of degree $n$ over the finite field $\mathbb{F}_{q}$ with $q$ elements. It is known that irreducible constituents of supercharacters partition the set of all irreducible characters $\operatorname{Irr}\left(U_{n}(q)\right)$. In this paper we present a correspondence between supercharacters and pattern subgroups of the form $U_{k}(q) \cap{ }^{w} U_{k}(q)$ where $w$ is a monomial matrix in $\operatorname{GL}_{k}(q)$ for some $k<n$.
\end{abstract}

\section{INTRODUCTION}

Let $q$ be a power of a prime $p$ and $\mathbb{F}_{q}$ a field with $q$ elements. The group $U_{n}(q)$ of all upper triangular $(n \times n)$-matrices over $\mathbb{F}_{q}$ with all diagonal entries equal to 1 is a Sylow $p$-subgroup of $\mathrm{GL}_{n}\left(\mathbb{F}_{q}\right)$. It is conjectured by Higman [8] that the number of conjugacy classes of $U_{n}(q)$ is given by a polynomial in $q$ with integer coefficients. Isaacs [10] shows that the degrees of all irreducible characters of $U_{n}(q)$ are powers of $q$. Huppert [9] proves that character degrees of $U_{n}(q)$ are precisely of the form $\left\{q^{e}: 0 \leq e \leq \mu(n)\right\}$ where the upper bound $\mu(n)$ was known to Lehrer [14]. Lehrer [14] conjectures that each number $N_{n, e}(q)$ of irreducible characters of $U_{n}(q)$ of degree $q^{e}$ is given by a polynomial in $q$ with integer coefficients. Isaacs [11] suggests a strengthened form of Lehrer's conjecture stating that $N_{n, e}(q)$ is given by a polynomial in $(q-1)$ with nonnegative integer coefficients. So, Isaacs' Conjecture implies Higman's and Lehrer's Conjectures.

Many efforts have been made to understand more about $U_{n}(q)$, see $[1,3,5$, $7,10,11,16,17,18]$, among others. Supercharacters arise as tensor products of some elementary characters to give a 'nice' partition of all non-principal irreducible characters of $U_{n}(q)$, see $[1,13]$. Supercharacters have been defined for Sylow $p$ subgroups of other finite groups of Lie type (see [2]), and in general for finite algebra groups (see [5]).

Here, for $U_{n}(q)$ we show a natural correspondence between supercharacters and pattern subgroups (Theorem 2.8). To highlight the main idea of construction, we have deferred all of our proofs to Section 3. Next we apply this correspondence to decompose certain supercharacters into irreducible constituents in the last section.

\section{Suppercharacters AND PATtERN SUBGroups}

Let $\Sigma:=\Sigma_{n-1}=\left\langle\alpha_{1}, \ldots, \alpha_{n-1}\right\rangle$ be the root system of $\mathrm{GL}_{n}(q)$ with respect to the maximal split torus equal to the diagonal group, see [4, Chapter 3]. Denote $\alpha_{i, j}:=\alpha_{i}+\alpha_{i+1}+\ldots+\alpha_{j}$ for all $0<i \leq j<n$. Denote by $\Sigma^{+}$the set of all positive

Date: March 2nd, 2010.

2010 Mathematics Subject Classification. Primary 20C15, 20D15. Secondary 20C33, 20D20.

Key words and phrases. Root system, irreducible character, triangular group. 
roots. The root subgroup $X_{\alpha_{i, j}}$ is the set of all matrices of the form $I_{n}+c \cdot e_{i, j+1}$, where $I_{n}=$ the identity $(n \times n)$-matrix, $c \in \mathbb{F}_{q}$ and $e_{i, j+1}=$ the zero matrix except a ' 1 ' at entry $(i, j+1)$. The upper triangular group $U_{n}(q)$ is generated by all $X_{\alpha}$ where $\alpha \in \Sigma^{+}$. We write $U$ for $U_{n}(q)$ if $n$ and $q$ are clear from the context. For convenience when using the root system, we consider the upper triangular group as a tableaux.

$$
\left(\begin{array}{ccccc}
1 & * & * & * & * \\
\cdot & 1 & * & * & * \\
\cdot & \cdot & 1 & * & * \\
\cdot & \cdot & \cdot & 1 & * \\
\cdot & \cdot & \cdot & \cdot & 1
\end{array}\right) \longrightarrow \begin{array}{cc|c|c|c|}
\hline \alpha_{1} & \alpha_{1,2} & \alpha_{1,3} & \alpha_{1,4} \\
\hline & & \alpha_{2} & \alpha_{2,3} & \alpha_{2,4} \\
\hline
\end{array}
$$

A subset $S \subset \Sigma^{+}$is called closed if for each $\alpha, \beta \in S$ such that $\alpha+\beta \in \Sigma^{+}$then $\alpha+\beta \in S$. A pattern subgroup of $U$ is a group generated by all root subgroups $X_{\alpha}$, where $\alpha \in S$ a closed positive root subset.

Let $G$ be a group. Denote $G^{\times}:=G-\{1\}, \operatorname{Irr}(G)$ the set of all complex irreducible characters of $G$, and $\operatorname{Irr}(G)^{\times}:=\operatorname{Irr}(G)-\left\{1_{G}\right\}$. For $H \unlhd G$, let $\operatorname{Irr}(G / H)$ denote the set of all irreducible characters of $G$ with $H$ in the kernel. If $K \leq G$ such that $G=H \rtimes K$, then for each character $\xi$ of $K$, we denote the inflation of $\xi$ to $G$ by $\xi_{G}$, i.e. $\xi_{G}$ is the extension of $\xi$ to $G$ with $H \subset \operatorname{ker}\left(\xi_{G}\right)$. Furthermore, for $H \leq G$ and $\xi \in \operatorname{Irr}(H)$, we define $\operatorname{Irr}(G, \xi):=\left\{\chi \in \operatorname{Irr}(G):\left(\chi, \xi^{G}\right) \neq 0\right\}$ the irreducible constituent set of $\xi^{G}$, and for $\chi \in \operatorname{Irr}(G)$, we denote its restriction to $H$ by $\left.\chi\right|_{H}$.

For a field $K$, let $K^{\times}:=K-\{0\}$. In the whole paper, we fix a nontrivial linear character $\varphi: \mathbb{F}_{q} \rightarrow \mathbb{C}^{\times}$. For each $\alpha \in \Sigma^{+}$and $s \in \mathbb{F}_{q}$, the map $\phi_{\alpha, s}: X_{\alpha} \rightarrow \mathbb{C}^{\times}$, $x_{\alpha}(d) \mapsto \varphi(d s)$ is a linear character of $X_{\alpha}$, and all linear characters of $X_{\alpha}$ arise in this way.

For each $\alpha_{i, j}$, we define

$$
\operatorname{arm}\left(\alpha_{i, j}\right):=\left\{\alpha_{i, k}: i \leq k<j\right\} \text { and } \operatorname{leg}\left(\alpha_{i, j}\right):=\left\{\alpha_{k, j}: i<k \leq j\right\} .
$$

If $i=j, \alpha_{i, i}=\alpha_{i}$, then $\operatorname{arm}\left(\alpha_{i}\right)$ and $\operatorname{leg}\left(\alpha_{i}\right)$ are empty. For each $\alpha \in \Sigma^{+}$, we define the hook of $\alpha$ as $h(\alpha):=\operatorname{arm}(\alpha) \cup \operatorname{leg}(\alpha) \cup\{\alpha\}$, the hook group of $\alpha$ as $H_{\alpha}:=\left\langle X_{\beta}: \beta \in h(\alpha)\right\rangle$, and the base group $V_{\alpha}:=\left\langle X_{\beta}: \beta \in \Sigma^{+}-\operatorname{arm}(\alpha)\right\rangle$. Since $\left[V_{\alpha}, V_{\alpha}\right] \cap X_{\alpha}=\{1\}$, for each $s \in \mathbb{F}_{q}^{\times}$there exists a linear $\lambda_{\alpha, s} \in \operatorname{Irr}\left(V_{\alpha}\right)$ such that $\left.\lambda_{\alpha, s}\right|_{X_{\alpha}}=\phi_{\alpha, s}$ and $\left.\lambda_{\alpha, s}\right|_{X_{\beta}}=1_{X_{\beta}}$ for the others $X_{\beta} \subset V_{\alpha}, \beta \neq \alpha$. Denote by $\operatorname{Irr}\left(V_{\alpha} /\left[V_{\alpha}, V_{\alpha}\right]\right)^{\times}$the set of all these linear characters of $V_{\alpha}$.

Lemma 2.1. $\lambda_{\alpha, s}^{U}$ is irreducible for all $s \in \mathbb{F}_{q}^{\times}$.

Proof. See [1, Lemma 2] or [13, Lemma 2.2].

We call $\lambda_{\alpha, s}^{U}$ an elementary character of $U$ associated to $\alpha$. A basic set $D$ is a nonempty subset of $\Sigma^{+}$in which none of roots are on the same row or column. For each basic set $D$, we define $E(D):=\bigoplus_{\alpha \in D} \operatorname{Irr}\left(V_{\alpha} /\left[V_{\alpha}, V_{\alpha}\right]\right)^{\times}$.

For each basic set $D$ and $\phi \in E(D)$, we define a supercharacter, also known as basic character in [1],

$$
\xi_{D, \phi}:=\bigotimes_{\lambda_{\alpha, s} \in \phi} \lambda_{\alpha, s}{ }^{U}
$$

It turns out that each supercharacter $\xi_{D, \phi}$ is induced from a linear character of a pattern subgroup. 
Definition 2.2. We define $V_{D}:=\bigcap_{\alpha \in D} V_{\alpha}$ and $\lambda_{D}:=\left.\bigotimes_{\lambda_{\alpha, s} \in \phi} \lambda_{\alpha, s}\right|_{V_{D}}$.

Lemma 2.3. We have $\xi_{D, \phi}=\lambda_{D}^{U}$.

Proof. See [13, Lemma 2.5].

It is easy to see that $V_{D}$ is generated by all $X_{\beta}$ where $\beta \in \Sigma^{+}-\left(\bigcup_{\alpha \in D} \operatorname{arm}(\alpha)\right)$, and $\lambda_{D}$ is a linear character of $V_{D}$. For each basic set $D$, it can be proven that the diagonal subgroup of $\mathrm{GL}_{n}(q)$ acts transitively on $E(D)$ by conjugation. So it makes sense when we write $\lambda_{D}$ here instead of $\lambda_{D, \phi}$, and it also says that the decomposition of $\xi_{D, \phi}$ is dependent only on $D$. To know more about supercharacters, see [5, 6]. Here, we recall the main role of supercharacters as a partition of $\operatorname{Irr}(U)^{\times}$.

Theorem 2.4. For each $\chi \in \operatorname{Irr}(U)^{\times}$, there exist uniquely a basic set $D$ and $\phi \in E(D)$ such that $\chi$ is an irreducible constituent of $\xi_{D, \phi}$.

Proof. See [1, Theorem 1] or [13, Theorem 2.6].

Denote by $\operatorname{Irr}\left(\xi_{D, \phi}\right)$ the set of all irreducible constituents of $\xi_{D, \phi}$. Here, to prove Higman's Conjecture, it suffices to prove that $\left|\operatorname{Irr}\left(\xi_{D, \phi}\right)\right|$ is a polynomial in $q$.

Now for each basic set $D$ of size $k=|D|$, we define an associated monomial $(k \times k)$-matrix $w_{D} \in \mathrm{GL}_{k}(q)$. First of all, we define two partial orders on $\Sigma^{+}$.

Definition 2.5. We define $<_{r}$ and $<_{b}$ on $\Sigma^{+}$as follows

(i) $\alpha_{i, j}<_{r} \alpha_{l, k}$ if $j<k$ (i.e. to the right)

(ii) $\alpha_{i, j}<_{b} \alpha_{l, k}$ if $i<l$ (i.e. to the bottom).

An easy way to understand these two orders is $<_{r}$ standing for left to right and $<_{b}$ for top to bottom. Notice that on a basic set, $<_{r}$ and $<_{b}$ are total orders.

Now we fix a basic set $D$ of size $k$ ascending order of $<_{r}$. Let $D:=\left\{\tau_{1}, . ., \tau_{k}\right\}$ where $\tau_{i}<_{r} \tau_{j}$ if $i<j$. We define $w_{D}:=\left(a_{i, j}\right) \in \mathrm{GL}_{k}(q)$ as follows

$$
a_{i, j}:= \begin{cases}1 & \text { if } \tau_{j} \text { is the } i \text {-th element of } D \text { in ascending order }<_{b}, \\ 0 & \text { otherwise. }\end{cases}
$$

For example, if $D:=\left\{\alpha_{2,3}, \alpha_{1,4}, \alpha_{3,5}\right\},|D|=3$,

\begin{tabular}{|l|l|l|l|l|}
\hline & & $\alpha_{1,4}$ & \\
\hline & $\alpha_{2,3}$ & & \\
\hline & & & $\alpha_{3,5}$ \\
\cline { 2 - 3 } & & & \\
\hline
\end{tabular} then $w_{D}=\left(\begin{array}{lll}0 & 1 & 0 \\
1 & 0 & 0 \\
0 & 0 & 1\end{array}\right)$.

It is clear that $w_{D}$ is a monomial matrix in the Weyl group $S_{k}$ of $\mathrm{GL}_{k}(q)$. Here, $w_{D}$ somehow gives pivots of $D$ by considering only rows and columns containing roots in $D$. Hence, it is equivalent to apply the (total) orders $<_{r},<_{b}$ to these monomial matrices on their nonzero entries.

For each pair $0<i<j \leq k$, if $\tau_{i}<_{b} \tau_{j}$, let $\gamma_{i, j}$ be the root on the row of $\tau_{i}$ such that $\gamma_{i, j}+\tau_{j} \in \Sigma^{+}$; otherwise, i.e. $\tau_{j}<_{b} \tau_{i}$, let $\nu_{i, j}$ be the root on the row of $\tau_{j}$ such that $\nu_{i, j}+\tau_{i} \in \Sigma^{+}$. For example, $\tau_{i}:=\alpha_{m, i}, \tau_{j}:=\alpha_{l, j}$ where $i<j$, so if $\alpha_{m, i}<_{b} \alpha_{l, j}$, i.e. $m<l$, then $\gamma_{i, j}=\alpha_{m, l-1}$; otherwise, if $\alpha_{l, j}<_{b} \alpha_{m, i}$, i.e. $l<m$, then $\nu_{i, j}=\alpha_{l, m-1}$. It is easy to see that $\nu_{i, j}$ exists if and only if two hooks $h\left(\tau_{i}\right)$ and $h\left(\tau_{j}\right)$ are parallel, otherwise $\gamma_{i, j}$ exists (Figure 1 ). 




Figure 1. Positions of $\nu_{i, j}$ and $\gamma_{i, j}$

Let $\Gamma_{D}$ be the set of all $\gamma_{i, j}$, let $\Lambda_{D}$ be the set of all $\nu_{i, j}$, and $\Delta_{D}:=\Gamma_{D} \cup \Lambda_{D}$. By definitions for the existence of $\gamma_{i, j}$ and $\nu_{i, j}$, we have $\Gamma_{D} \cap \Lambda_{D}=\emptyset$.

Definition 2.6. We define $R_{D}:=\left\langle X_{\gamma}: \gamma \in \Gamma_{D}\right\rangle$ and $C_{D}:=\left\langle X_{\nu}: \nu \in \Lambda_{D}\right\rangle$.

The next lemma provides interesting correspondences between the size of $D$ and $\Delta_{D}$, and between $w_{D}$ and $\Gamma_{D}, \Lambda_{D}$. Moreover, it shows that $\left\langle V_{D}, R_{D}\right\rangle=V_{D} R_{D}$, and the pattern subgroups $R_{D}, C_{D}$ are only determined by $w_{D}$ in a natural way.

Lemma 2.7. Let $D$ be a basic set of size $k$. The following are true.

(i) $\Delta_{D}$ is closed and $\left\langle X_{\alpha}: \alpha \in \Delta_{D}\right\rangle$ is isomorphic to $U_{k}(q)$.

(ii) $\Gamma_{D}$ is closed. For each pair $i<j$, if $\gamma_{i, s}, \gamma_{j, r}$ exist and $\gamma_{i, s}+\gamma_{j, r} \in \Sigma^{+}$, then $s=j$ and $\gamma_{i, j}+\gamma_{j, r}=\gamma_{i, r}$.

(iii) $\Lambda_{D}$ is closed. For each pair $i<j$, if $\nu_{i, s}, \nu_{j, r}$ exist and $\nu_{i, s}+\nu_{j, r} \in \Sigma^{+}$, then $s=j$ and $\nu_{i, j}+\nu_{j, r}=\nu_{i, r}$.

(iv) $R_{D}$ is isomorphic to $U_{k}(q) \cap{ }^{w_{D}} U_{k}(q)$ and $C_{D}$ is isomorphic to $U_{k}(q) \cap w_{0} w_{D} U_{k}(q)$ where $w_{0}$ is the longest element in the Weyl group $S_{k}$ of $\mathrm{GL}_{k}(q)$, also known as

$$
w_{0}=\left(\begin{array}{ccc}
0 & \cdots & 1 \\
\vdots & . & \vdots \\
1 & \cdots & 0
\end{array}\right) .
$$

(v) $V_{D} R_{D}$ is a pattern subgroup of $U$ and $R_{D}$ normalizes $V_{D}$.

For example, let $D:=\left\{\alpha_{1,2}, \alpha_{3,4}, \alpha_{4,5}, \alpha_{2,6}\right\}$ be a basic set in $\Sigma_{6}^{+}$.

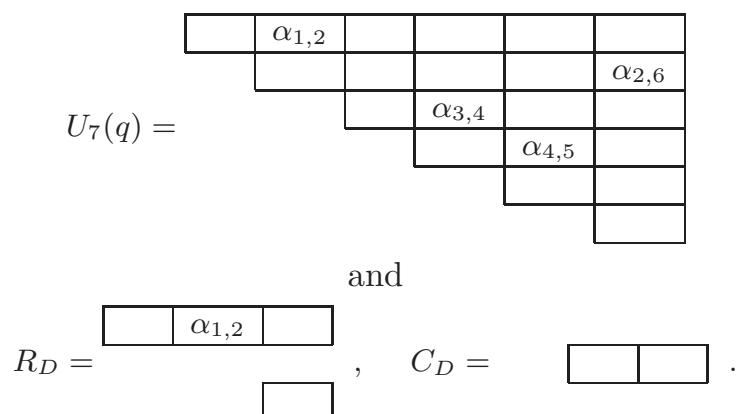

The next result is the main theorem, which provides a correspondence between supercharacters $\xi_{D, \phi}$ and pattern subgroups $R_{D}$.

Theorem 2.8. Let $\xi_{D, \phi}$ be a supercharacter. The following are true. 
(i) $\xi_{D, \phi}=\left(\lambda_{D}^{V_{D} R_{D}}\right)^{U}$.

(ii) For each $\chi \in \operatorname{Irr}\left(V_{D} R_{D}, \lambda_{D}\right), \chi^{U} \in \operatorname{Irr}\left(\xi_{D, \phi}\right)$.

(iii) If $\chi_{1} \neq \chi_{2} \in \operatorname{Irr}\left(V_{D} R_{D}, \lambda_{D}\right)$, then $\chi_{1}^{U} \neq \chi_{2}^{U}$.

Therefore, to decompose $\xi_{D, \phi}$, it suffices to decompose $\lambda_{D}^{V_{D} R_{D}}$. Moreover, the induced character $\lambda_{D}^{V_{D} R_{D}}$ is equal to $\left(\left.\lambda_{D}\right|_{V_{D} \cap R_{D}}{ }^{R_{D}}\right)_{V_{D} R_{D}} \otimes \theta$, where $\theta$ is some linear character of $V_{D} R_{D}$ (in Lemma 3.1). We see that $\left.\lambda_{D}\right|_{V_{D} \cap R_{D}}{ }^{R_{D}}$ is a 'very special' constituent of the regular character $1^{R_{D}}$. Hence, the decomposition method of all supercharacters $\xi_{D, \phi}$ of $U_{n}(q)$ with the same $w_{D}$ is generally restricted to the one of the regular character $1^{R_{D}}$.

Here, we attempt to make a link for this special pattern $R_{D}=U_{k}(q) \cap^{w_{D}} U_{k}(q)$ in Lemma 2.7. Denote $U \cap{ }^{w} U$ by $U_{w}$, where $U=U_{n}(q)$ and $w \in S_{n}$ is the Weyl group of $\mathrm{GL}_{n}(q)$, Thompson [18] conjectured that for each pair $r, s \in S_{n}$, the cardinality of the double coset $U_{r} \backslash U / U_{s}$ is a polynomial in $q$ with integer coefficients. In addition, $U_{w}$ also takes an important role when one studies $\mathrm{GL}_{n}(q)$ as groups with $(B, N)$-pair, such as the Bruhat decomposition.

From Theorem 2.8 and Lemma 2.7 (v), we obtain a nice decomposition of $\xi_{D, \phi}$.

Corollary 2.9. Let $\xi_{D, \phi}$ be a supercharacter. The following are tru:

(i) $\operatorname{Irr}\left(\xi_{D, \phi}\right)=\left\{\chi^{U}: \chi \in \operatorname{Irr}\left(V_{D} R_{D}, \lambda_{D}\right)\right\}$,

(ii) $\xi_{D, \phi}=\sum_{\chi \in \operatorname{Irr}\left(V_{D} R_{D}, \lambda_{D}\right)} \chi(1) \chi^{U}$.

Theorem 2.4, Lemma 2.7 and Corollary 2.9 give a clear proof for the following corollary, which is a different version of [1, Theorem 1.4].

Corollary 2.10. $\left(\xi_{D, \phi}, \xi_{D^{\prime}, \phi^{\prime}}^{\prime}\right)= \begin{cases}\left|V_{D} R_{D}: V_{D}\right| & \text { if }(D, \phi)=\left(D^{\prime}, \phi^{\prime}\right), \\ 0 & \text { otherwise. }\end{cases}$

As an application to $U_{13}(q)$, we answer the conjecture by Isaacs-Karagueuzian [12] that $U_{13}(2)$ has a unique pair of irrational irreducible characters of degree $2^{16}$. This conjecture is solved with an affirmative answer by Marberg [15] and generalized by Evseev [7]. Here, we work independently to obtain representations and constructions of all irreducible constituents of the supercharacter of $U_{13}(q)$, which gives the irrational pair when $q=2$. With the definition that a character is well-induced if it is induced from a linear character of some pattern subgroup by Evseev [7], these two irrational characters are not well-induced. Thus, it provides a more explainable script to the generalization of not well-induced characters. Finally, we list two families of supercharacters $\xi_{D, \phi}$ which have exactly one irreducible constituent, i.e. $\xi_{D, \phi}=m \chi$ for some $\chi \in \operatorname{Irr}(U)$.

\section{All proofs}

Here we mainly prove Theorem 2.8 to give a natural correspondence between supercharacters $\xi_{D, \phi}$ and pattern subgroups $U_{k}(q) \cap{ }^{w_{D}} U_{k}(q)$ where $k=|D|$. First, we shall prove Lemma 2.7.

Proof of Lemma 2.7. Suppose that $D:=\left\{\tau_{1}, \ldots, \tau_{k}\right\}$ in ascending order $<_{r}$.

(i) If we rearrange $D$ in ascending order of $<_{b}$ to be $\left\{\theta_{1}, . ., \theta_{k}\right\}$, then on the row of $\theta_{i}, \Delta_{D}$ has $(k-i)$ roots and the row of $\theta_{k}$ does not have any root in $\Delta_{D}$.

For each pair $i<j \in[1, k]$, let $\omega_{i, j} \in \Delta_{D}$ be the root on the row of $\tau_{i}$ such that $\omega_{i, j}+\tau_{j} \in \Sigma^{+}$. (Notice that $\omega_{i, j}$ is either $\gamma \in \Gamma_{D}$ or $\nu \in \Lambda_{D}$.) Hence, if $\tau_{i}=\alpha_{i_{1}, i_{2}}<_{b} \tau_{j}=\alpha_{j_{1}, j_{2}}$, i.e. $i_{1}<j_{1}$, we have $\omega_{i, j}=\alpha_{i_{1}, j_{1}-1}$. Therefore, for each 
$\omega_{i, j}=\alpha_{i_{1}, j_{1}-1}<_{r} \omega_{m, l}=\alpha_{m_{1}, l_{1}-1} \in \Delta_{D}$, if $\omega_{i, j}+\omega_{m, l} \in \Sigma^{+}$, then it must be $j_{1}=m_{1}$, and $\omega_{i, j}+\omega_{j, l}=\alpha_{i_{1}, l_{1}-1}=\omega_{i, l}$. This shows that $\Delta_{D}$ is closed, and the longest root in $\Delta_{D}$ is $\omega_{1,2}+\ldots+\omega_{k-1, k}=\omega_{1, k}$. So $\omega_{i, j}$ corresponds to $\alpha_{i, j-1}$ in the positive root set $\Sigma_{k-1}^{+}$. Therefore, $\left\langle X_{\alpha}: \alpha \in \Delta_{D}\right\rangle$ is a pattern subgroup isomorphic to $U_{k}(q)$.

(ii) With the same argument as in (i), by definitions of $\gamma_{i, s}, \gamma_{j, r}$, if $\gamma_{i, s}+\gamma_{j, r} \in \Sigma^{+}$, then $s=j$. By the transitivity of $<_{r},<_{b}$ on $\tau_{i}, \tau_{j}, \tau_{r}$, from $\tau_{i}<_{r},<_{b} \tau_{j}$ and $\tau_{j}<_{r},<_{b} \tau_{k}$, we have $\tau_{i}<_{r},<_{b} \tau_{r}$. So $\gamma_{i, r}$ exists and $\gamma_{i, j}+\gamma_{j, r}=\gamma_{i, r}$ follows.

(iii) The same argument of (ii) holds for $\nu_{i, s}$ and $\nu_{j, r} \in \Lambda_{D}$.

(iv) Let $w_{D}:=\left(w_{i, j}\right) \in S_{k} \subset \operatorname{GL}_{k}(q)$. Since $w_{D}$ is a monomial matrix, $w_{D}^{-1}=w_{D}^{T}$, the transpose of $w_{D}$. For each $X=\left(x_{i, j}\right) \in U_{k}(q)$, we observe $Y:=w_{D} \cdot X \cdot w_{D}{ }^{-1}$. Let $Y=\left(y_{i, j}\right)$. For each pair $i<j$, we have $y_{i, j}=\sum_{s, r \in[1, k]} w_{i, s} x_{s, r} w_{j, r}$. Since $i, j$ are fixed, there exist uniquely $1 \leq f, h \leq k$ such that $w_{i, f}=1=w_{j, h}$, others $w_{i, s}=0=w_{j, r}$. Hence, $y_{i, j}=w_{i, f} x_{f, h} w_{j, h}$.

Since $h \neq f$ and all $x_{s, r}=0$ if $r<s$, we have the following

- $y_{i, j}=0$ if $f>h$, i.e. $w_{i, f}<_{b} w_{j, h}$ and $w_{j, h}<_{r} w_{i, f}$;

- $y_{i, j} \neq 0$ if $f<h$, i.e. $w_{i, f}<_{b} w_{j, h}$ and $w_{i, f}<_{r} w_{j, h}$.

So $R_{D}$ is isomorphic to $U_{k}(q) \cap{ }^{w_{D}} U_{k}(q)$ by the definition of $\gamma_{i, j} \in \Gamma_{D}$. And, hence, $C_{D}$ is isomorphic to $U_{k}(q) \cap{ }^{w_{0}} \cdot w_{D} U_{k}(q)$ by (i), (ii), (iii) and $\Delta_{D}=\Gamma_{D} \cup \Lambda_{D}$.

(v) From the definition of $\gamma_{i, j}$, it is easy to check that $R_{D}$ normalizes $V_{D}$. Hence, $V_{D} R_{D}$ is a pattern subgroup of $U$.

Set $K_{D}:=\left\langle X_{\alpha}: X_{\alpha} \subset V_{D}\right.$ and $\left.\alpha \notin D\right\rangle=\left\langle X_{\alpha}: X_{\alpha} \subset V_{D} \cap \operatorname{ker}\left(\lambda_{D}\right)\right\rangle$. It is clear that $K_{D}$ is normal in $V_{D},\left[V_{D}: K_{D}\right]=q^{|D|}$, and $V_{D}=K_{D} \cdot \prod_{\tau \in D} X_{\tau}$. To prove Theorem 2.8, we need the following lemma.

Lemma 3.1. Let $\xi_{D, \phi}$ be a supercharacter. The following are true.

(i) $K_{D} \subset \operatorname{ker}\left(\lambda_{D}^{V_{D} R_{D}}\right)$. Moreover, $\lambda_{D}^{V_{D} R_{D}}(x)=\left|V_{D} R_{D}: V_{D}\right| \lambda_{D}(x)$ for all $x \in V_{D}$.

(ii) $\left(K_{D} \cap R_{D}\right) \unlhd R_{D}$ and $\left(V_{D} \cap R_{D}\right) /\left(K_{D} \cap R_{D}\right) \subset Z\left(R_{D} /\left(K_{D} \cap R_{D}\right)\right)$.

(iii) Let $\bar{\phi}_{D}:=\left\{\lambda_{\alpha, s} \in \phi: X_{\alpha} \nsubseteq R_{D}\right\}$. We have

$$
\lambda_{D}^{V_{D} R_{D}}=\left(\left.\lambda_{D}\right|_{V_{D} \cap R_{D}} ^{R_{D}}\right)_{V_{D} R_{D}} \otimes\left(\bigotimes_{\lambda_{\alpha, s} \in \bar{\phi}_{D}}\left(\left.\lambda_{\alpha, s}\right|_{V_{D}}\right)_{V_{D} R_{D}}\right) .
$$

Proof. (i) It suffices to show the statement for all $X_{\alpha} \subset V_{D}$. By Lemma 2.7 (v) $V_{D} \unlhd V_{D} R_{D}$, for all $x \in V_{D}$ we have

$$
\lambda_{D}^{V_{D} R_{D}}(x)=\frac{1}{\left|V_{D}\right|} \sum_{y \in V_{D} R_{D}} \lambda_{D}\left(x^{y}\right) .
$$

For each $x \in X_{\alpha}$, we suppose that there is $X_{\beta} \subset V_{D} R_{D}$ such that $\alpha+\beta \in \Sigma^{+}$, hence $X_{\alpha+\beta} \subset V_{D}$. We shall show that $\lambda_{D}\left(x^{y}\right)=\lambda_{D}(x)$ for all $y \in X_{\beta}$.

Since $X_{\tau} \cap\left[V_{D}, V_{D}\right]=\{1\}$ for all $\tau \in D$, we have $X_{\alpha+\beta} \subset K_{D} \subset \operatorname{ker}\left(\lambda_{D}\right)$. Thus, $\left[\lambda_{D}(x), \lambda_{D}(y)\right]=\lambda_{D}([x, y])=1$ since $[x, y] \in X_{\alpha+\beta}$, i.e. $\lambda_{D}(x)^{-1} \lambda_{D}\left(x^{y}\right)=1$.

(ii) By the definition of $K_{D} \unlhd V_{D}$ and $V_{D}=K_{D} \cdot \prod_{\tau \in D} X_{\tau}$, it suffices to show that $\left(K_{D} \cap R_{D}\right) \unlhd R_{D}$. This is clear because for all $X_{\alpha} \subset K_{D} \cap R_{D}$ and all $X_{\beta} \subset R_{D}$, either $\alpha+\beta \notin \Sigma^{+}$or $X_{\alpha+\beta} \subset K_{D} \cap R_{D}$. 
(iii) The inflations to $V_{D} R_{D}$ of $\left.\lambda_{D}\right|_{V_{D} \cap R_{D}}{ }^{R_{D}}$ and $\left.\lambda_{\alpha, s}\right|_{V_{D}}$, for all $\lambda_{\alpha, s} \in \bar{\phi}_{D}$, follow directly from (i).

Remark. By Lemma 3.1 (iii), if $R_{D} \cap V_{D}=\{1\}$, then $\lambda_{D}^{V_{D} R_{D}}$ is equivalent to the regular character $1^{R_{D}}$ of $R_{D}$. In general, $\lambda_{D}^{V_{D} R_{D}}$ is equivalent to a constituent of $1^{R_{D}}$ with $R_{D} \cap K_{D}$ in the kernel. Now we prove Theorem 2.8.

Proof of Theorem 2.8. (i) This is clear by the transitivity of induction.

(ii) We suppose that $D:=\left\{\tau_{1}, \ldots, \tau_{k}\right\}$ in ascending order $<_{r}$ and for $s_{i} \in \mathbb{F}_{q}^{\times}$let $\lambda_{D}:=\left.\bigotimes_{\tau_{i} \in D} \lambda_{\tau_{i}, s_{i}}\right|_{V_{D}}$.

First, we show that for each $\chi \in \operatorname{Irr}\left(V_{D} R_{D}, \lambda_{D}\right), \chi^{U}$ is irreducible. By the transitivity of induction, we shall induce $\chi$ from $V_{D} R_{D}$ to $U$ by a sequence of inductions along the arms of $\tau_{1}, \tau_{2}, \ldots, \tau_{k}$ respectively by $<_{r}$ order. Now we setup these such induction steps.

For each $\tau_{i} \in D$, let $A\left(\tau_{i}\right)=\left\{\alpha \in \operatorname{arm}\left(\tau_{i}\right): X_{\alpha} \nsubseteq V_{D} R_{D}\right\}$, and $c_{i}=\left|A\left(\tau_{i}\right)\right|$. Let $d_{0}=0$ and $d_{i}=d_{i-1}+c_{i}$ for all $i \in[1, k]$. Now if $c_{i}>0, i \in[1, k]$, we arrange $A\left(\tau_{i}\right)$ in decreasing order $<_{r}$ to be $\left\{\beta_{d_{i-1}+1}, \ldots, \beta_{d_{i-1}+c_{i}}\right\}$. Let $M_{0}=V_{D} R_{D}$, $M_{i+1}=M_{i} \rtimes X_{\beta_{i}}$ for all $i \in\left[1, d_{k}\right]$. It is clear that $M_{d_{k}+1}=U$ and $X_{\beta_{j}}$ normalizes $M_{j}$; hence, this sequence of pattern subgroups is well-defined.

For each $\beta_{j} \in \operatorname{arm}\left(\tau_{i}\right), j \in\left[1, d_{k}\right]$, there exists uniquely $\delta \in \operatorname{leg}\left(\tau_{i}\right)$ such that $\beta_{j}+\delta=\tau_{i}$ and $X_{\delta} \subset K_{D}$, since if $X_{\delta} \nsubseteq K_{D}$, there exists $\tau_{m} \in D$ such that $\delta \in \operatorname{arm}\left(\tau_{m}\right)$, so $\tau_{i}<_{r} \tau_{m}, \tau_{i}<_{b} \tau_{m}$, and this implies $\beta_{j}=\gamma_{i, m}$. We number this $\delta$ as $\delta_{j}$, and let $L(D):=\left\{\delta_{j}: j \in\left[1, d_{k}\right]\right\}$. By Lemma 3.1 (i), $X_{\delta} \subset \operatorname{ker}(\chi)$ for all $\delta \in L(D)$. Now we proceed the induction of $\chi$ from $V_{D} R_{D}$ to $U$ via a sequence of pattern subgroups along the arms of all $\tau_{i} \in D$, namely from $M_{0}$ to $M_{1}, \ldots$, $M_{d_{k}+1}=U$.

Suppose that $\chi^{M_{j}} \in \operatorname{Irr}\left(M_{j}\right)$ for some $M_{j}, j \in\left[1, d_{k}+1\right]$ and $X_{\delta_{t}} \subset \operatorname{ker}\left(\chi^{L}\right)$ for all $t \in\left[j, d_{k}\right]$. If $j=d_{k}+1$, the process is complete. Otherwise, the next induction step is from $M_{j}$ to $M_{j+1}=M_{j} X_{\beta_{j}}$, and we suppose that it happens on the arm of $\tau_{i}$. For each $x \in X_{\beta_{j}}^{\times}$, since $\left[X_{\delta_{j}}, x\right]=X_{\tau_{i}}$, there is some $y \in X_{\delta_{j}}$ such that $\lambda_{\tau_{i}, s_{i}}([y, x]) \neq 1$ and

$$
{ }^{x}\left(\chi^{M_{j}}\right)(y)=\chi^{M_{j}}\left(y^{x}\right)=\chi^{M_{j}}([y, x] y)=\lambda_{\tau_{i}, s_{i}}([y, x]) \chi^{M_{j}}(y) \neq \chi^{M_{j}}(y)=\chi^{M_{j}}(1) .
$$

Hence, $X_{\delta_{j}} \nsubseteq \operatorname{ker}\left({ }^{x}\left(\chi^{M_{j}}\right)\right)$, and ${ }^{x}\left(\chi^{M_{j}}\right) \neq \chi^{M_{j}}$ for all $x \in X_{\beta_{j}}^{\times}$. This shows that the inertia group $I_{M_{j} X_{\beta_{j}}}(\chi)=M_{j}$ and $\chi^{M_{j} X_{\beta_{j}}} \in \operatorname{Irr}\left(M_{j} X_{\beta_{j}}, \lambda_{D}\right)$.

It is easy to check directly that $X_{\delta_{t}} \subset \operatorname{ker}\left(\chi^{M_{j} X_{\beta_{j}}}\right)$ for all $t \in\left[j+1, d_{k}\right]$ by using $\left[X_{\beta_{j}}, X_{\delta_{t}}\right] \subset \operatorname{ker}\left(\chi^{M_{j}}\right)$. Therefore, $\chi^{U}$ is irreducible for all $\chi \in \operatorname{Irr}\left(V_{D} R_{D}, \lambda_{D}\right)$ by induction on $j$.

(iii) Now suppose that $\chi_{1} \neq \chi_{2} \in \operatorname{Irr}\left(V_{D} R_{D}, \lambda_{D}\right)$ and $\chi_{1}{ }^{M_{j}} \neq \chi_{2}{ }^{M_{j}}$ for some $M_{j}$. As above, it is enough to show that $\chi_{1}^{M_{j} X_{\beta_{j}}} \neq \chi_{2}^{M_{j} X_{\beta_{j}}}$, where $\beta_{j} \in \operatorname{arm}\left(\tau_{i}\right)$. Notice that $X_{\delta_{j}} \subset \operatorname{ker}\left(\chi_{1}{ }^{M_{j}}\right) \cap \operatorname{ker}\left(\chi_{2}{ }^{M_{j}}\right)$.

By Mackey formula with the double coset $M_{j} \backslash M_{j} X_{\beta_{j}} / M_{j}$ represented by $X_{\beta_{j}}$, $\left(\chi_{1}{ }^{M_{j} X_{\beta_{j}}}, \chi_{2}{ }^{M_{j} X_{\beta_{j}}}\right)=\sum_{x \in X_{\beta_{j}}}\left(\chi_{1}{ }^{M_{j}},{ }^{x}\left(\chi_{2}{ }^{M_{j}}\right)\right)$. Using the same argument as in (ii), we have $X_{\delta_{j}} \nsubseteq \operatorname{ker}\left({ }^{x}\left(\chi_{2}{ }^{M_{j}}\right)\right)$ for all $x \in X_{\beta_{j}}^{\times}$. Hence, ${ }^{x}\left(\chi_{2}{ }^{M_{j}}\right) \neq \chi_{1}{ }^{M_{j}}$ for all

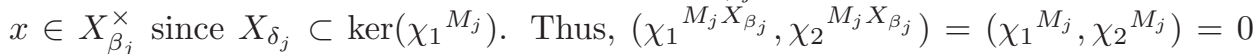
since $\chi_{1}{ }^{M_{j}} \neq \chi_{2}{ }^{M_{j}}$ by the above assumption on $M_{j}$. 
Notice that $V_{D} R_{D}$ is not normal in $U$. In the proof of Theorem 2.8, although all inductions from $V_{D} R_{D}$ to $U$ are irreducible, Clifford correspondence can not be applied. The technique of a sequence of inductions from $M_{j}$ to $M_{j+1} \subset N_{U}\left(M_{j}\right)$ has been used to control distinct induced characters.

Since $V_{D}$ is normal in $V_{D} R_{D}$ and $V_{D} R_{D} / V_{D} \cong R_{D} /\left(V_{D} \cap R_{D}\right)$, by Theorem 2.8 and Lemma 3.1 (iii), we only need to decompose $\left.\lambda_{D}\right|_{V_{D} \cap R_{D}} R_{D}$ instead of decomposing the supercharacter $\xi_{D, \phi}=\lambda_{D}^{U}$. Hence, all work is restricted to a pattern subgroup of $U_{k}(q)$ where $k=|D|<n$.

Proof of Corollary 2.9. Theorem 2.8 provides a one-to-one correspondence on the multiplicities and degrees between two constituent sets $\operatorname{Irr}\left(V_{D} R_{D}, \lambda_{D}\right)$ and $\operatorname{Irr}\left(\xi_{D, \phi}\right)$, i.e. $\left|\operatorname{Irr}\left(V_{D} R_{D}, \lambda_{D}\right)\right|=\left|\operatorname{Irr}\left(\xi_{D, \phi}\right)\right|$, and if $\chi \in \operatorname{Irr}\left(V_{D} R_{D}, \lambda_{D}\right)$ has multiplicity $t$ then $\chi^{U} \in \operatorname{Irr}\left(\xi_{D, \phi}\right)$ also has multiplicity $t$, and $\chi^{U}(1)=\left|U: V_{D} R_{D}\right| \chi(1)$. Therefore, it is enough to show that $\chi \in \operatorname{Irr}\left(R_{D},\left.\lambda_{D}\right|_{V_{D} \cap R_{D}}\right)$ has multiplicity $\chi(1)$.

By Lemma 3.1 (i), $K_{D} \cap V_{D} \subset \operatorname{ker}\left(\left.\lambda_{D}\right|_{V_{D} \cap R_{D}}\right) \cap \operatorname{ker}\left(\left.\lambda_{D}\right|_{V_{D} \cap R_{D}} R_{D}\right)$ is normal in $R_{D}$. So $\left.\lambda_{D}\right|_{V_{D} \cap R_{D}}$ can be considered as a linear character of the quotient group $R_{D} /\left(K_{D} \cap R_{D}\right)$. By Lemma 3.1 (ii), $\left(V_{D} \cap R_{D}\right) /\left(K_{D} \cap R_{D}\right) \subset Z\left(R_{D} /\left(K_{D} \cap R_{D}\right)\right)$, $\left.\lambda_{D}\right|_{V_{D} \cap R_{D}}$ is a linear character of the center, and the claim holds.

\section{Applichtions}

Example 4.1. Here we list two families of supercharacters $\xi_{D, \phi}$ of $U_{n}(q)$ which have only one irreducible constituent, i.e. $\left|\operatorname{Irr}\left(\xi_{D, \phi}\right)\right|=1$. Without loss of generality, we suppose that $\alpha_{1,-} \in D$.

$D_{1}:=\left\{\alpha_{1, k}, \alpha_{2,2 k-1}, \alpha_{3,2 k-2}, \ldots, \alpha_{k, k+1}, \alpha_{k+1,2 k}\right\}$ where $4 \leq 2 k<n$. We have

$$
w_{D_{1}}=\left(\begin{array}{ccc}
1 & \cdot & \cdot \\
\cdot & w & \cdot \\
\cdot & \cdot & 1
\end{array}\right)
$$

where $w$ is the longest element in the Weyl group $S_{k-1}$ of $\mathrm{GL}_{k-1}(q)$.

$D_{2}:=\left\{\alpha_{1,2}, \alpha_{2,3}, \ldots, \alpha_{2 m-1,2 m}\right\}$ where $2 \leq 2 m<n$, which gives $w_{D_{2}}$ equal to the identity $(2 m-1) \times(2 m-1)$-matrix $I_{2 m-1}$.

By Lemma 2.7, $R_{D_{1}}$ is the largest special subgroup $q^{1+2(k-1)}$ in $U_{k+1}(q)$, i.e. $\left[R_{D_{1}}, R_{D_{1}}\right]=Z\left(R_{D_{1}}\right)=\Phi\left(R_{D_{1}}\right)$ the Frattini subgroup of $R_{D_{1}}$. It is known that a special subgroup of type $q^{1+2 t}$ has $q^{2 t}$ linear characters and $q-1$ almost faithful irreducible characters of degree $q^{t}$, see [13, Corollary 2.3]. (Recall that $\chi \in \operatorname{Irr}(G)$ is almost faithful if $Z(G) \not \subset \operatorname{ker}(\chi)$.) Since $V_{D_{1}} \cap R_{D_{1}}=X_{\alpha_{1, k}}=Z\left(R_{D_{1}}\right)$, $\left.\lambda_{D_{1}}\right|_{X_{\alpha_{1, k}}} \neq 1_{X_{\alpha_{1, k}}}$. Hence, $\lambda_{D_{1}}^{V_{D_{1}} R_{D_{1}}}$ has only one irreducible constituent of degree $q^{k-1}$ with multiplicity $q^{k-1}$. By Corollary $2.9, \xi_{D_{1}, \phi}$ has only one constituent of degree $q^{(k-1)+\left|U: V_{D_{1}} R_{D_{1}}\right|}$ with multiplicity $q^{k-1}$.

We decompose $\xi_{D_{2}, \phi}$ by induction on $m$. By Corollary 2.9 and Lemma 3.1, we have $\left|\operatorname{Irr}\left(\xi_{D_{2}, \phi}\right)\right|=\left|\operatorname{Irr}\left(R_{D_{2}},\left.\lambda_{D_{2}}\right|_{V_{D_{2}} \cap R_{D_{2}}}\right)\right|$. Let $D_{2}^{\prime}:=\left\{\alpha_{1,2}, \alpha_{2,3}, \ldots, \alpha_{2 m-3,2 m-2}\right\}$. Since $w_{D_{2}}=I_{2 m-1}$, by Lemma 2.7, $R_{D_{2}} \simeq U_{2 m-1}(q)$. It is easy to check that $D_{2}^{\prime}=\left\{\alpha \in D: X_{\alpha} \subset R_{D_{2}}\right\}$ and $\left.\lambda_{D_{2}}\right|_{V_{D_{2}} \cap R_{D_{2}}}{ }^{R_{D_{2}}}=q \xi_{D_{2}^{\prime}, \phi^{\prime}}$ where $\xi_{D_{2}^{\prime}, \phi^{\prime}}$ is a supercharacter of $U_{2 m-1}(q)$ with $\phi^{\prime}=\phi-\left\{\lambda_{\alpha_{2 m-2,2 m-1}, s_{2 m-2}}, \lambda_{\alpha_{2 m-1,2 m}, s_{2 m-1}}\right\}$. Hence, by the hypothesis of induction on $m$, it suffices to check $D_{2}=\left\{\alpha_{1,2}, \alpha_{2,3}, \alpha_{3,4}\right\}$, which is $D_{1}$ with $k=2$. 
Example 4.2. Isaacs and Karagueuzian [12] conjectured that $U_{13}(2)$ has a unique pair of irrational irreducible characters of degree $2^{16}$. This conjecture was answered by Marberg ([15, Theorem 9.2]). By constructing decomposition trees, he also found out the exact supercharacter whose irreducible constituent set contains this pair. Evseev ([7, Theorem 1.4]) generalized the result by applying a reduction algorithm process to obtain $q(q-1)^{13}$ irreducibles of $U_{13}(q)$ which cannot be constructed by inducing from a linear character of some pattern subgroup. Those characters are called not well-induced. We work independently and point out the supercharacter with these properties as well.

To deal with extensions of linear characters from a subgroup, we use the following property whose proof is quite obvious.

Lemma 4.3. For $H \leq G$ and $\lambda \in \operatorname{Irr}(H)$, if $[G, G] \leq \operatorname{ker}(\lambda)$ then $\lambda$ extends to $G$.

Let $U:=U_{13}(q)$ and $D:=\left\{\alpha_{1,4}, \alpha_{2,5}, \alpha_{3,9}, \alpha_{4,10}, \alpha_{5,6}, \alpha_{6,7}, \alpha_{7,8}, \alpha_{8,11}, \alpha_{9,12}\right\} \subset \Sigma_{12}^{+}$. By Lemma 2.7, we have

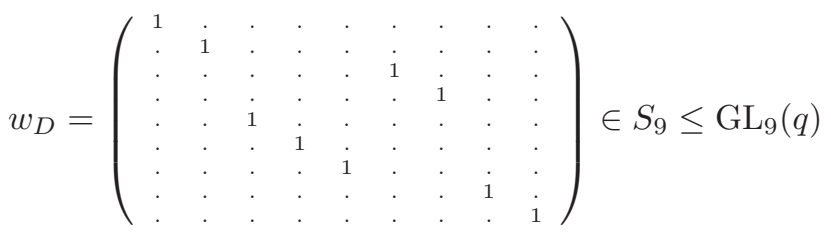

and

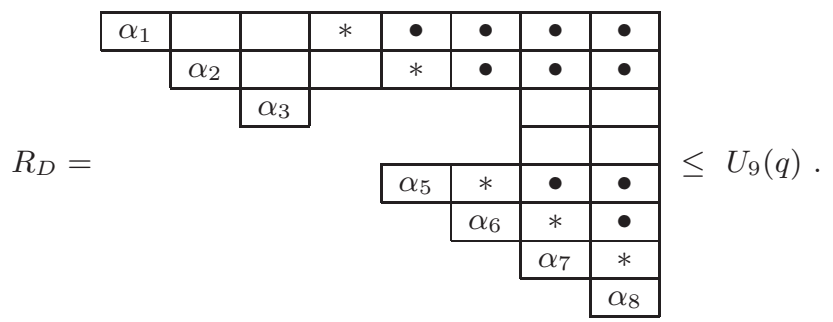

As above $\left|U: V_{D} R_{D}\right|=q^{12}, \lambda_{D}^{U}(1)=q^{27}, R_{D}=U_{9}(q) \cap w_{D} U_{9}(q), D \cap \Gamma_{D}=$ all *'s, $K_{D} \cap R_{D}=$ all $X_{\bullet}$ 's $\subset \operatorname{ker}\left(\lambda_{D}\right)$, and $V_{D} \cap R_{D}=$ both $X_{*}$ 's and $X_{\bullet}$ 's. Precisely, let $\mu:=\left.\lambda_{D}\right|_{V_{D} \cap R_{D}}$. Then $\left.\mu\right|_{X_{\tau_{i}}}=\phi_{\tau_{i}, s_{i}}$ where $\tau_{i} \in\left\{\alpha_{1,4}, \alpha_{2,5}, \alpha_{5,6}, \alpha_{6,7}, \alpha_{7,8}\right\}=*^{\prime} s$ and $s_{i} \in \mathbb{F}_{q}^{\times}$. Notice that $\mu$ is a linear character of $R_{D} \cap V_{D} \subset U_{9}(q)$.

Since $K_{D} \cap R_{D} \subset \operatorname{ker}(\mu) \cap \operatorname{ker}\left(\mu^{R_{D}}\right)$, we proceed the induction of $\mu$ in the quotient group $R_{D} /\left(K_{D} \cap R_{D}\right)$. Let $R:=R_{D} /\left(K_{D} \cap R_{D}\right)$ and $V:=\left(V_{D} \cap R_{D}\right) /\left(K_{D} \cap R_{D}\right)$. Abusing terminology we call the image of a root subgroup of $U$ under the natural projection to its factor group by a pattern subgroup, a root subgroup.

To obtain all constituents $\chi$ of $\mu^{R}$, we use the following strategy. We start with the factor group $R / H$ where $H$ is the largest pattern subgroup contained in $\operatorname{ker}(\chi)$. Let $Q \leq R / H$ such that $Q$ is maximal in the set of all pattern subgroups $P$ of $R / H$ satisfying $[P, P] \leq \operatorname{ker}(\mu)$. By Lemma $4.3, \mu$ extends to $Q$. Let $\lambda$ be an extension of $\mu$ to $Q$. In most of the cases, $Q \triangleleft R / H$ and $I_{R / H}(\lambda)=Q$ except the subcase e.

Proposition 4.4. $\mu^{R}$ decomposes into

- $q^{3}(2 q-1)$ irreducibles of degree $q^{3}$, each has multiplicity $q^{3}$;

- $q(q-1)\left(3 q^{3}+q^{2}+q-1\right)$ irreducibles of degree $q^{4}$, each has multiplicity $q^{4}$;

- $q^{2}(q+2)(q-1)^{2}$ irreducibles of degree $q^{5}$, each has multiplicity $q^{5}$. 
Proof. Since $X_{\alpha_{1,3}} X_{\alpha_{3,8}} \subset Z(R), \mu^{L_{1}}$ splits into $q^{2}$ linears of $L_{1}:=V X_{\alpha_{1,3}} X_{\alpha_{3,8}}$. Let $\lambda_{1}$ be an extension of $\mu$ to $L_{1}$. We divide the proof into four cases:

Case 1: $\lambda_{1}\left(X_{\alpha_{1,3}}\right) \neq\{1\} \neq \lambda_{1}\left(X_{\alpha_{3,8}}\right)$, there are $(q-1)^{2}$ such $\lambda_{1}$ 's.

Case 2: $\lambda_{1}\left(X_{\alpha_{1,3}}\right) \neq\{1\}=\lambda_{1}\left(X_{\alpha_{3,8}}\right)$, there are $(q-1)$ such $\lambda_{1}$ 's.

Case 3: $\lambda_{1}\left(X_{\alpha_{1,3}}\right)=\{1\} \neq \lambda_{1}\left(X_{\alpha_{3,8}}\right)$, there are $(q-1)$ such $\lambda_{1}$ 's.

Case 4: $\lambda_{1}\left(X_{\alpha_{1,3}}\right)=\{1\}=\lambda_{1}\left(X_{\alpha_{3,8}}\right)$, there is only one such $\lambda_{1}$.

Case 1: Let $L_{2}:=L_{1} X_{\alpha_{1,2}} X_{\alpha_{2}} X_{\alpha_{2,3}} X_{\alpha_{2,4}} X_{\alpha_{6}} X_{\alpha_{3,7}} X_{\alpha_{4,7}} X_{\alpha_{4,8}}$. Check that $L_{2} \unlhd R,\left[L_{2}, L_{2}\right] \leq \operatorname{ker}\left(\lambda_{1}\right)$ and $R=L_{2} X_{\alpha_{1}} X_{\alpha_{3}} X_{\alpha_{5}} X_{\alpha_{7}} X_{\alpha_{8}}$. So $\lambda_{1}^{L_{2}}$ splits into $q^{8}$ linear characters of $L_{2}$. Let $\lambda_{2}$ be an extension of $\lambda_{1}$ to $L_{2}$. Check that the inertia group $I_{R}\left(\lambda_{2}\right)=L_{2}$ and, by Clifford theory, $\lambda_{2}^{R} \in \operatorname{Irr}(R)$ has degree $q^{5}$. So we obtain $(q-1)^{2} q^{3}$ irreducible constituents of degree $q^{5}$, each has multiplicity $q^{5}$.

Remark. To avoid more notations, we still denote by $R$ its quotient groups $R / H$ where $H$ is some normal pattern subgroup of $R$ in the coming proofs.

Case 2: Here $X_{\alpha_{3,8}} \subset \operatorname{ker}\left(\lambda_{1}^{R}\right)$, we work with $R / X_{\alpha_{3,8}}$. Since $X_{\alpha_{4,8}} \subset Z(R), \lambda_{1}$ extends to $H_{2}:=L_{1} X_{\alpha_{4,8}}$. Let $\eta_{2}$ be an extension of $\lambda_{1}$ to $H_{2}$.

If $\eta_{2}\left(X_{\alpha_{4,8}}\right) \neq\{1\}$, then let $H_{3}:=H_{2} X_{\alpha_{2}} X_{\alpha_{6}} X_{\alpha_{8}} X_{\alpha_{1,2}} X_{\alpha_{2,3}} X_{\alpha_{2,4}} X_{\alpha_{3,7}}$. Check that $\left[H_{3}, H_{3}\right] \leq \operatorname{ker}\left(\eta_{2}\right), H_{3} \unlhd R$ and $R=H_{3} X_{\alpha_{1}} X_{\alpha_{3}} X_{\alpha_{5}} X_{\alpha_{7}} X_{\alpha_{4,7}}$. So $\eta_{2}^{H_{3}}$ splits into $q^{7}$ linear characters. Call $\eta_{3}$ an extension of $\eta_{2}$ to $H_{3}$. Check that $I_{R}\left(\eta_{3}\right)=H_{3}$ and $\eta_{3}^{R} \in \operatorname{Irr}(R)$. Thus there are $(q-1)^{2} q^{2}$ irreducible constituents of degree $q^{5}$, each has multiplicity $q^{5}$.

Otherwise, if $\eta_{2}\left(X_{\alpha_{4,8}}\right)=\{1\}$, then $X_{\alpha_{4,8}} \subset \operatorname{ker}\left(\eta_{2}{ }^{R}\right)$. We work with $R / X_{\alpha_{4,8}}$. Let $H_{3}:=H_{2} X_{\alpha_{2}} X_{\alpha_{6}} X_{\alpha_{8}} X_{\alpha_{1,2}} X_{\alpha_{2,3}} X_{\alpha_{2,4}} X_{\alpha_{3,7}} X_{\alpha_{4,7}}$. Check that $\left[H_{3}, H_{3}\right] \leq \operatorname{ker}\left(\eta_{2}\right)$, $H_{3} \unlhd R$ and $R=H_{3} X_{\alpha_{1}} X_{\alpha_{3}} X_{\alpha_{5}} X_{\alpha_{7}}$. So $\eta_{2}^{H_{3}}$ splits into $q^{8}$ linear characters. Call $\eta_{3}$ an extension of $\eta_{2}$ to $H_{3}$. Check that $I_{R}\left(\eta_{3}\right)=H_{3}$ and $\eta_{3}^{R} \in \operatorname{Irr}(R)$. Thus, there are $(q-1) q^{4}$ irreducible constituents of degree $q^{4}$, each has multiplicity $q^{4}$.

Case 3: Here $X_{\alpha_{1,3}} \subset \operatorname{ker}\left(\lambda_{1}^{R}\right)$, we work with $R / X_{\alpha_{1,8}}$. Since $X_{\alpha_{1,2}} \subset Z(R), \lambda_{1}$ extends to $N_{2}:=L_{1} X_{\alpha_{1,2}}$. Call $\lambda_{2}$ an extension of $\lambda_{1}$ to $N_{2}$.

If $\lambda_{2}\left(X_{\alpha_{1,2}}\right) \neq\{1\}$, let $N_{3}:=N_{2} X_{\alpha_{1}} X_{\alpha_{5}} X_{\alpha_{7}} X_{\alpha_{2,3}} X_{\alpha_{3,7}} X_{\alpha_{4,7}} X_{\alpha_{4,8}}$. Check that $\left[N_{3}, N_{3}\right] \leq \operatorname{ker}\left(\lambda_{2}\right), N_{3} \unlhd R$ and $R=N_{3} X_{\alpha_{2}} X_{\alpha_{3}} X_{\alpha_{6}} X_{\alpha_{8}} X_{\alpha_{2,4}}$. So $\lambda_{2}^{N_{3}}$ splits into $q^{7}$ linear characters. Let $\lambda_{3}$ be an extension of $\lambda_{2}$ to $N_{3}$. Check that $I_{R}\left(\lambda_{3}\right)=N_{3}$ and $\lambda_{3}^{R} \in \operatorname{Irr}(R)$. Thus there are $(q-1)^{2} q^{2}$ irreducible constituents of degree $q^{5}$, each has multiplicity $q^{5}$.

Otherwise, if $\lambda_{2}\left(X_{\alpha_{1,2}}\right)=\{1\}$, then $X_{\alpha_{1,2}} \subset \operatorname{ker}\left(\lambda_{2}\right)$. We work with $R / X_{\alpha_{1,2}}$. Let $N_{3}:=N_{2} X_{\alpha_{1}} X_{\alpha_{2}} X_{\alpha_{5}} X_{\alpha_{7}} X_{\alpha_{2,3}} X_{\alpha_{3,7}} X_{\alpha_{4,7}} X_{\alpha_{4,8}}$. Check that $\left[N_{3}, N_{3}\right] \leq \operatorname{ker}\left(\lambda_{2}\right)$, $R=N_{3} X_{\alpha_{3}} X_{\alpha_{6}} X_{\alpha_{8}} X_{\alpha_{2,4}}$ and $N_{3} \unlhd R$. So $\lambda_{2}^{N_{3}}$ splits into $q^{8}$ linear characters. Call $\lambda_{3}$ an extension of $\lambda_{2}$ to $N_{3}$. Check that $I_{R}\left(\lambda_{3}\right)=N_{3}$ and $\lambda_{3}^{R} \in \operatorname{Irr}(R)$. Thus there are $(q-1) q^{4}$ irreducible constituents of degree $q^{4}$, each has multiplicity $q^{4}$.

Case 4: Here $X_{\alpha_{1,3}} X_{\alpha_{3,8}} \subset \operatorname{ker}\left(\lambda_{1}^{R}\right)$, we work with $R / X_{\alpha_{1,3}} X_{\alpha_{3,8}}$. Since $X_{\alpha_{1,2}}$ $X_{\alpha_{2,3}} X_{\alpha_{3,7}} X_{\alpha_{4,8}} \subset Z(R), \lambda_{1}$ extends to $T_{2}:=L_{1} X_{\alpha_{1,2}} X_{\alpha_{2,3}} X_{\alpha_{3,7}} X_{\alpha_{4,8}}=Z(R)$. Let $\mu_{2}$ be an extension of $\lambda_{1}$ to $T_{2}$. Notice that there are nine pattern special $p$ groups $S_{i}$ of type $q^{1+2}$ whose centers are contained in $Z(R)$; for each $X_{\alpha} \subset R-Z(R)$, there exists exactly a pair $S_{i} \neq S_{j}$ such that $S_{i} \cap S_{j}=X_{\alpha}$.

$$
\begin{array}{lll}
S_{1}=X_{\alpha_{1}} X_{\alpha_{1,2}} X_{\alpha_{2}}, & S_{2}=X_{\alpha_{2}} X_{\alpha_{2,3}} X_{\alpha_{3}}, & S_{3}=X_{\alpha_{3}} X_{\alpha_{3,7}} X_{\alpha_{4,7}} \\
S_{4}=X_{\alpha_{4,7}} X_{\alpha_{4,8}} X_{\alpha_{8}}, & S_{5}=X_{\alpha_{8}} X_{\alpha_{7,8}} X_{\alpha_{7}}, & S_{6}=X_{\alpha_{7}} X_{\alpha_{6,7}} X_{\alpha_{6}}, \\
S_{7}=X_{\alpha_{6}} X_{\alpha_{5,6}} X_{\alpha_{5}}, & S_{8}=X_{\alpha_{5}} X_{\alpha_{2,5}} X_{\alpha_{2,4}}, & S_{9}=X_{\alpha_{2,4}} X_{\alpha_{1,4}} X_{\alpha_{1}}
\end{array}
$$


We shall study five subcases based on which of the four root subgroups $X_{\alpha_{1,2}}$, $X_{\alpha_{2,3}}, X_{\alpha_{3,7}}, X_{\alpha_{4,8}}$ are contained in $\operatorname{ker}\left(\mu_{2}\right)$.

Subcase a: All are contained in $\operatorname{ker}\left(\mu_{2}\right)$. Let $T_{3}:=T_{2} X_{\alpha_{2}} X_{\alpha_{3}} X_{\alpha_{4,7}} X_{\alpha_{1}} X_{\alpha_{5}} X_{\alpha_{7}}$. Since $\left[T_{3}, T_{3}\right] \leq \operatorname{ker}\left(\mu_{2}\right), \mu_{2}$ extends to $T_{3}$. Call $\mu_{3}$ an extension of $\mu_{2}$ to $T_{3}$. Each $\mu_{3}$ induces irreducibly to $R$ by checking $I_{R}\left(\mu_{3}\right)=T_{3}$. Thus, there are $q^{3}$ irreducible constituents of degree $q^{3}$, each has multiplicity $q^{3}$.

Subcase b: Three of them are in $\operatorname{ker}\left(\mu_{2}\right)$ and one is not. There are $\left(\begin{array}{l}4 \\ 1\end{array}\right)=4$ smaller cases corresponding to each root subgroup $\not \subset \operatorname{ker}\left(\mu_{2}\right)$. Let $\bar{R}$ denote the factor group of $R$ by the three root subgroups $\subset \operatorname{ker}\left(\mu_{2}\right)$. They fall into two families where $|Z(\bar{R})|=q^{8}$ or $q^{7}$. If either $X_{\alpha_{1,2}}$ or $X_{\alpha_{4,8}} \not \subset \operatorname{ker}\left(\mu_{2}\right)$, then $|Z(\bar{R})|=q^{8}$ and $\mu_{2}^{\bar{R}}$ has $q^{3}(q-1)$ irreducible constituents of degree $q^{3}$, each has multiplicity $q^{3}$. Otherwise, if either $X_{\alpha_{2,3}}$ or $X_{\alpha_{3,7}} \not \subset \operatorname{ker}\left(\mu_{2}\right)$, then $|Z(\bar{R})|=q^{7}$ and $\mu_{2}^{\bar{R}}$ has $q(q-1)$ irreducible constituents of degree $q^{4}$, each has multiplicity $q^{4}$. We shall decompose $\mu_{2}^{R}$ for one case of each type, the others are similar.

If $X_{\alpha_{1,2}} \not \subset \operatorname{ker}\left(\mu_{2}\right)$, let $T_{3}:=T_{2} X_{\alpha_{3}} X_{\alpha_{4,7}} X_{\alpha_{2}} X_{\alpha_{2,4}} X_{\alpha_{6}} X_{\alpha_{8}}$. If $X_{\alpha_{2,3}} \not \subset \operatorname{ker}\left(\mu_{2}\right)$, let $T_{3}:=T_{2} X_{\alpha_{4,7}} X_{\alpha_{2}} X_{\alpha_{2,4}} X_{\alpha_{6}} X_{\alpha_{8}}$. Since $\left[T_{3}, T_{3}\right] \leq \operatorname{ker}\left(\mu_{2}\right), \mu_{2}$ extends to $T_{3}$. Call $\mu_{3}$ an extension of $\mu_{2}$ to $T_{3}$. Then $\mu_{3}^{R} \in \operatorname{Irr}(R)$ by checking $I_{R}\left(\mu_{3}\right)=T_{3}$.

So we obtain $2(q-1) q^{3}$ irreducible constituents of degree $q^{3}$, each has multiplicity $q^{3}$, and $2(q-1) q$ irreducible constituents of degree $q^{4}$, each has multiplicity $q^{4}$.

Subcase c: Two of them are in $\operatorname{ker}\left(\mu_{2}\right)$ and the others are not. There are $\left(\begin{array}{l}4 \\ 2\end{array}\right)=6$ smaller cases. Applied the same argument, in each case $\mu_{2}^{R}$ has $q(q-1)^{2}$ irreducible constituents of degree $q^{4}$, each has multiplicity $q^{4}$. Thus, we obtain $6(q-1)^{2} q$ irreducible constituents of degree $q^{4}$, each has multiplicity $q^{4}$.

Subcase d: One is in $\operatorname{ker}\left(\mu_{2}\right)$ and the other three are not. There are 4 smaller cases. WLOG, we suppose $X_{\alpha_{1,2}} \subset \operatorname{ker}\left(\mu_{2}\right)$. Let $T_{3}:=T_{2} X_{\alpha_{1}} X_{\alpha_{5}} X_{\alpha_{7}} X_{\alpha_{4,7}} X_{\alpha_{2}}$. Since $\left[T_{3}, T_{3}\right] \leq \operatorname{ker}\left(\mu_{2}\right), \mu_{2}$ extends to $T_{3}$. Call $\mu_{3}$ an extension of $\mu_{2}$. Then $\mu_{3}^{R} \in \operatorname{Irr}(R)$ by checking $I_{R}\left(\mu_{3}\right)=T_{3}$. So there are $4(q-1)^{3} q$ irreducible constituents of degree $q^{4}$, each has multiplicity $q^{4}$.

Subcase e: None of them is in $\operatorname{ker}\left(\mu_{2}\right)$. Let $Q_{3}:=T_{2} X_{\alpha_{1}} X_{\alpha_{5}} X_{\alpha_{7}} X_{\alpha_{4,7}} \unlhd R$. Since $\left[Q_{3}, Q_{3}\right] \leq \operatorname{ker}\left(\mu_{2}\right), \mu_{2}$ extends to $Q_{3}$. Call $\mu_{3}$ an extension of $\mu_{2}$ to $Q_{3}$. Let $\left.\mu_{3}\right|_{X_{\beta_{i}}}=\phi_{\beta_{i}, s_{i}}$ where $\beta_{i} \in\left\{\alpha_{1,2}, \alpha_{2,3}, \alpha_{3,7}, \alpha_{4,8}, \alpha_{7,8}, \alpha_{6,7}, \alpha_{5,6}, \alpha_{2,5}, \alpha_{1,4}\right\}$ and $s_{i} \in \mathbb{F}_{q}^{\times}$. The inertia group $I_{R}\left(\mu_{3}\right)$ is generated by $Q_{3}$ and $x(a)$ for all $a \in \mathbb{F}_{q}$ where

$$
x(a):=x_{\alpha_{2}}\left(-\frac{s_{1,4}}{s_{1,2}} a\right) x_{\alpha_{6}}\left(\frac{s_{2,5}}{s_{5,6}} a\right) x_{\alpha_{8}}\left(\frac{s_{2,5} s_{6,7}}{s_{5,6} s_{7,8}} a\right) x_{\alpha_{3}}\left(\frac{s_{2,5} s_{4,8} s_{6,7}}{s_{3,7} s_{5,6} s_{7,8}} a\right) x_{\alpha_{2,4}}(a) .
$$

We claim $\left|I_{R}\left(\mu_{3}\right): Q_{3}\right|=q$ and $\left[I_{R}\left(\mu_{3}\right), I_{R}\left(\mu_{3}\right)\right] \leq \operatorname{ker}\left(\mu_{3}\right)$ by showing that $\left[x_{\beta}(c), x(a)\right] \in \operatorname{ker}\left(\mu_{3}\right)$ where $\beta \in\left\{\alpha_{1}, \alpha_{5}, \alpha_{7}, \alpha_{4,7}\right\}$ and $c, a \in \mathbb{F}_{q}$. By the nilpotent class 2 of $R,[x, y z]=[x, z][x, y]^{z}=[x, z][x, y]$. E.g. with $\beta=\alpha_{1}$, we have

$$
\begin{aligned}
{\left[x_{\alpha_{1}}(c), x(a)\right] } & =\left[x_{\alpha_{1}}(c), x_{\alpha_{2,4}}(a)\right]\left[x_{\alpha_{1}}(c), x_{\alpha_{2}}\left(-\frac{s_{1,4}}{s_{1,2}} a\right)\right] \\
& =x_{\alpha_{1,4}}(a c) x_{\alpha_{1,2}}\left(-\frac{s_{1,4}}{s_{1,2}} a c\right) .
\end{aligned}
$$

Therefore,

$$
\begin{aligned}
\mu_{3}\left(\left[x_{\alpha_{1}}(c), x(a)\right]\right) & =\mu_{3}\left(x_{\alpha_{1,4}}(a c) x_{\alpha_{1,2}}\left(-\frac{s_{1,4}}{s_{1,2}} a c\right)\right) \\
& =\phi_{\alpha_{1,4}, s_{1,4}}\left(x_{\alpha_{1,4}}(a c)\right) \phi_{\alpha_{1,2}, s_{1,2}}\left(x_{\alpha_{1,2}}\left(-\frac{s_{1,4}}{s_{1,2}} a c\right)\right) \\
& =\phi\left(s_{1,4} a c-s_{1,2} \frac{s_{1,4}}{s_{1,2}} a c\right)=1 .
\end{aligned}
$$


So $\mu_{3}$ extends to $I_{R}\left(\mu_{3}\right)$ and each extension $\mu_{4}$ induces irreducibly to $R$. Thus, there are $(q-1)^{4} q$ irreducible constituents of degree $q^{4}$, each has multiplicity $q^{4}$.

Remark. In the subcase e, $I_{R}\left(\mu_{3}\right)$ is not a pattern subgroup. By Theorem 2.8 $\xi_{D, \phi}=\lambda_{D}^{U}$ has $\left|\operatorname{Irr}\left(V_{D} R_{D}, \lambda_{D}\right)\right|=(q-1)^{4} q$ distinct constituents of degree $q^{4+12}=q^{16}$. Since $|D|=9$, there are $(q-1)^{9}$ such supercharacters. So we have $(q-1)^{13} q$ not wellinduced characters as stated in [7]. For $q=2^{f}, x(a)^{2}=x_{\alpha_{2,3}}\left(\frac{s_{1,4} s_{2,5} s_{4,8} s_{6,7}}{s_{1,2} s_{3,7} s_{5,6} s_{7,8}} a^{2}\right) \notin \operatorname{ker}\left(\mu_{4}\right)$ and the order $\mathrm{o}(x(a))=4$ for all $a \in \mathbb{F}_{q}^{\times}$. There is $a_{0} \in \mathbb{F}_{q}$ such that $\mu_{4}\left(x\left(a_{0}\right)^{2}\right)=-1$. Thus, $\mu_{4}\left(x\left(a_{0}\right)\right)= \pm i \in \mathbb{C}-\mathbb{R}$, i.e. $\mu_{4}$ is an irrational linear character of $I_{R}\left(\mu_{3}\right)$. This explains why $\mu_{4}^{R}$ remains irrational, and so does its corresponding character in $\operatorname{Irr}\left(\lambda_{D}^{U}\right)$. This gives a way to construct the pair of irrational irreducible characters of degree $2^{16}$ of $U_{13}(2)$ for Isaacs-Karagueuzian's Conjecture [12].

\section{ACKNOWLEDGEMENT}

The content of this paper was presented at Conference on Algebraic Topology, Group Theory and Representation Theory, dedicated to 60-th birthdays of Professor Ron Solomon and Professor Bob Oliver, which was held on the Isle of Skye in 2009. The author is grateful to the organizers of the conference.

\section{REFERENCES}

[1] C.A.M. André, Basic characters of the unitriangular group (for arbitrary primes), Proc. Amer. Math. Soc. 130 (2002), 1943-1954. 1, 2, 3, 5

[2] C.A.M. André and A.M. Neto, Supercharacters of finite unipotent groups of types $B_{n}, C_{n}$ and $D_{n}$, J. Algebra 305 (2006), 394-429. 1

[3] J.M. Arregi and A. Vera-López, Conjugacy classes in Sylow $p$-subgroups of GL( $n, q)$, J. Algebra 152 (1992), 1-19. 1

[4] R.W. Carter, Simple Groups of Lie Type, Wiley, London, 1972. 1

[5] P. Diaconis and I.M. Isaacs, Supercharacters and superclasses for algebra groups. Trans. Amer. Math. Soc. 360 (2008), 2359-2392. 1, 3

[6] P. Diaconis and N. Thiem, Supercharacter formulas for pattern groups, Trans. Amer. Math. Soc. 361 (2009), 3501-3533. 3

[7] A. Evseev, Reduction for characters of finite algebra groups, J. Algebra 325 (2011), 321-351. $1,5,9,12$

[8] G. Higman, Enumerating p-groups. I: Inequalities, Proc. London Math. Soc. 10 (1960), 24-30. 1

[9] B. Huppert, Character theory of finite groups, Walter de Gruyter, Berlin, 1998. 1

[10] I.M. Isaacs, Characters of groups associated with finite algebras, J. Algebra 177 (1995), 708730. 1

[11] I.M. Isaacs, Counting characters of upper triangular groups, J. Algebra 315 (2007), 698-719. 1

[12] I.M. Isaacs and D. Karagueuzian, Involutions and characters of upper triangular matrix groups, Mathematics of Computation 252 (2005), 2027-2033. 5, 9, 12

[13] T. Le, Counting irreducible representations of large degree of the upper triangular groups, J. Algebra, Vol. 324 (2010), 1803-1817. 1, 2, 3, 8

[14] G.I. Lehrer, Discrete series and the unipotent subgroup, Compos. Math. 28 (1974), 9-19. 1

[15] E. Marberg, Constructing modules of algebra groups, Honors thesis, Stanford University, 2008. 5,9

[16] G. Robinson, Counting conjugacy classes of unitriangular groups associated to finite dimensional algebras, J. Group Theory 1 (1998), 271-274. 1

[17] J. Thompson, $k\left(U_{n}\left(F_{q}\right)\right)$, http://www.math.ufl.edu/fac/thompson.html. 1

[18] J. Thompson, $\left(U_{s}(F), U_{r}(F)\right)$ double cosets in $U(F)$, http://ww. math.ufl.edu/fac/ thompson.html. 1, 5

E-mail address: 1ttung96@yahoo.com 